\title{
Electromagnetic Regulation of Cell Activity
}

\author{
Sarah A. Stanley ${ }^{1,2}$ and Jeffrey M. Friedman ${ }^{3,4}$ \\ ${ }^{1}$ Diabetes, Obesity and Metabolism Institute, Icahn School of Medicine at Mount Sinai, New York, \\ New York 10029 \\ ${ }^{2}$ Department of Neuroscience, Icahn School of Medicine at Mount Sinai, New York, New York 10029 \\ ${ }^{3}$ Laboratory of Molecular Genetics, Rockefeller University, New York, New York 10065 \\ ${ }^{4}$ Howard Hughes Medical Institute, New York, New York 10065 \\ Correspondence: friedj@mail.rockefeller.edu
}

The ability to observe the effects of rapidly and reversibly regulating cell activity in targeted cell populations has provided numerous physiologic insights. Over the last decade, a wide range of technologies have emerged for regulating cellular activity using optical, chemical, and, more recently, electromagnetic modalities. Electromagnetic fields can freely penetrate cells and tissue and their energy can be absorbed by metal particles. When released, the absorbed energy can in turn gate endogenous or engineered receptors and ion channels to regulate cell activity. In this manner, electromagnetic fields acting on external nanoparticles have been used to exert mechanical forces on cell membranes and organelles to generate heat and interact with thermally activated proteins or to induce receptor aggregation and intracellular signaling. More recently, technologies using genetically encoded nanoparticles composed of the iron storage protein, ferritin, have been used for targeted, temporal control of cell activity in vitro and in vivo. These tools provide a means for noninvasively modulating gene expression, intracellular organelles, such as endosomes, and whole-cell activity both in vitro and in freely moving animals. The use of magnetic fields interacting with external or genetically encoded nanoparticles thus provides a rapid noninvasive means for regulating cell activity.

$T_{h}$ ools that allow targeted control of cell activity have proven invaluable for understanding the role of defined cells in regulating biological processes. These technologies often combine engineered ion channels or receptors with external actuators such as light, ligands, or electromagnetic fields to regulate cell activity and function. Each technology has a distinct set of features that can be used to tailor their application to particular questions.

One of the most widely used tools for neuromodulation is optogenetics, the use of light- responsive ion channels, such as channel rhodopsin, expressed in defined cells to control their function (Kim et al. 2017). Channel rhodopsin, a modified light-gated channel from chlamydamonas (Nagel et al. 2003), is usually delivered to defined regions or cell populations in vivo, either by targeted injection of viral vectors or by mating Cre-expressing transgenic mice to other mice with Cre-dependent expression of channel rhodopsin (Wang et al. 2007). Channel rhodopsin and its derivatives are gated by light. The timescale for activation is very

Editors: Valentin A. Pavlov and Kevin J. Tracey

Additional Perspectives on Bioelectronic Medicine available at www.perspectivesinmedicine.org

Copyright (C) 2019 Cold Spring Harbor Laboratory Press; all rights reserved; doi: 10.1101/cshperspect.a034322

Cite this article as Cold Spring Harb Perspect Med 2019;9:a034322 
rapid, usually milliseconds, and this allows patterns of light to be used to deliver patterned neural activity such as burst firing (Boyden et al. 2005). Since its initial description, a wide range of modified channels have been developed that react to different wavelengths of light, over a range of timescales, can transiently or stably activate cells, can inhibit cell activity, and enable optogenetic control of intracellular signaling via light-activated, modified G-protein-coupled receptors (Zemelman et al. 2003; Zhang et al. 2007; Berndt et al. 2009; Packer et al. 2012). The constructs can be directed to defined cell populations and because there is minimal light scatter and tissue penetration (Melo et al. 2001), light can precisely target cell bodies or projections within anatomically defined areas (Petreanu et al. 2007). However, this also means that light needs to be delivered to deeper structures using a fiber optic implant. Only local populations adjacent to the optical fiber will receive sufficient light to regulate activity and the use of optogenetics to modulate the activity of cell populations dispersed over a wider region or in multiple sites is thus more challenging. Secure positioning of the optical fiber in freely moving animals can also be difficult in more mobile regions such as the spine or to modulate peripheral nerves. In addition, light needs to be delivered to the implanted fiber either via an optical cable that tethers the animal or a head-mounted light source (Dagnew et al. 2017). For some studies, tethering or handling the animal to attach a light source can interfere with the behavior being assessed. So, for some applications, alternative methods to regulate cell activity have potential advantages.

Chemogenetic tools are also widely used for activation or silencing of cell activity (Sternson and Roth 2014). Initially, chemogenetics used insect channels gated by ivermectin but more recently less toxic drugs have been used to modulate cell activity (Frazier et al. 2013). These technologies use ion channels (Lerchner et al. 2007; Magnus et al. 2011) or G-protein-coupled receptors (Nawaratne et al. 2008; Alexander et al. 2009) that are modified to respond to an otherwise inert compound. In some cases, the channels can be further engineered so that they no longer respond to their natural ligand. The modified channels or G-protein-coupled receptors are genetically targeted to defined tissues in vivo either by viral injection or crossing Cre-expressing transgenic mice with a transgenic mouse line in which expression of the chemogenetic tool is dependent on expression of the Cre recombinase (Alexander et al. 2009). The chemogenetic channel or receptor can then be activated by injection of its ligand allowing temporally controlled, targeted cell activation, or silencing. There is a growing range of chemogenetic tools: cation channels that gate sodium or calcium ion entry are used for cell activation while chloride channels can be used for cell silencing (Magnus et al. 2011). Similarly, G-protein-coupled receptors that are linked to $\mathrm{Gi}, \mathrm{Gq}$, or Gs can be used to activate or silence cells. In contrast to optogenetics that is limited to discrete anatomic regions by light dispersion, chemogenetic tools can be used to target dispersed cell populations. In addition, animals do not need to be tethered during assessment. Alternatively, either the channels or agonists can be delivered locally to provide anatomic specificity (Arenkiel et al. 2008). However, while the time course of activation using chemogenetics is rapid enough to unequivocally establish a biologic response, the time course is much slower than the millisecond activation of optogenetics. Thus, in some cases, correlating physiological changes to neural activity can be more challenging when using chemogenetics and it is not possible to effect changes in the pattern of neural activation (Nawaratne et al. 2008). The ligands need to be administered a short time before assessing their effects and, in some circumstances, the drug or their metabolites can alter the behavior or physiological role being examined as a result of offtarget effects. However, the durable effect of drugs makes chemogenetics especially well-suited for assessing the chronic effects of modulating cell activity. Overall, optogenetic and chemogenetic tools are suitable for a wide range of applications; however, alternative approaches that would allow the rapid regulation of cell activity in freely moving animals could prove useful in some experimental settings including clinical situations. Systems that use electromagnetic 
signals to regulate cell activity have many of these features and are reviewed below.

\section{ELECTROMAGNETIC CONTROL OF CELL ACTIVITY}

Electromagnetic fields can penetrate tissue and in birds and other animals will guide migration, although the precise mechanism is unknown (Freake et al. 2006). More recently, it has been shown that electromagnetic fields can be used to regulate the activity of cells in proximity to external metal nanoparticles (Huang et al. 2010; Chen et al. 2015), or that express genetically encoded intracellular metal nanoparticles (ferritin) (Stanley et al. 2015; Wheeler et al. 2016; Duret et al. 2017; Hutson et al. 2017). In both cases, the particles transduce electromagnetic fields that gate ion channels, although the field strength that is required is much larger than that required for magnetic sensing by biologic systems. Unlike other effectors such as light or ligands that need to be delivered directly via implants or injections, electromagnetic fields freely penetrate tissue with minimal absorption and can thus be delivered remotely (Stauffer et al. 1984). Indeed, magnetic fields of sufficient strength to gate the channels are in common usage for noninvasive imaging by magnetic resonance imaging (MRI) (Setsompop et al. 2016; Stanley et al. 2016). Electrogmagnetic fields are also used in clinical practice to program cardiac pacemakers (Halperin et al. 2008) and for the treatment of depression by transcranial magnetic stimulation (TMS) (Kedzior et al. 2015). The combination of electromagnetic fields and nanoparticles to modulate cell function may, in time, allow new bioelectronic therapeutic applications.

\section{EXTERNAL MAGNETIC PARTICLES TO EXERT A MECHANICAL FORCE}

\section{Mechanical Stimulation}

Many studies have used magnetic fields with magnetic nanoparticles (MNPs) attached to the cell membrane or to a specific protein to apply a mechanical force. Moreover, differences in the strength of the applied field and the specific properties of the particles allow carefully titrated and rapid changes in the applied force. These techniques were first used almost 70 years ago to assess the physical characteristics of cytoplasm, in particular its viscosity and elasticity (Crick and Hughes 1950). This and other early studies established the use of exposing magnetic particles to an external magnetic field in biologic systems to rapidly and reversibly apply a graded force. The method was also adapted, in some cases, to produce local changes in the force applied and the ability to apply high forces for bending or twisting (Crick and Hughes 1950).

In recent years, numerous studies have used MNPs and magnetic fields to control cell activity by targeting the particles to cell-surface receptors. The particles can be targeted by coating the particles with antibodies directed to cell-surface receptors (Stanley et al. 2012), or specific membrane proteins (Munshi et al. 2017), or by addition of specific ligands, such as biotin, to the nanoparticle surface that interacts with modified cell-surface receptors expressing the binding partner such as streptavidin (Seo et al. 2016). Magnetic particles of a Zn-doped ferrite core and plasmonic gold shell functionalized with benzylguanine were targeted to individual SNAP-tagged Notch receptors or E-cadherin (Fig. 1). An applied magnetic field localized the Notch receptors to a specific subcellular location and $9 \mathrm{pN}$ forces on the receptor-initiated receptor signaling. For E-cadherin, low forces $(2 \mathrm{pN})$ to cluster the molecule initiated F-actin assembly, which was then stabilized by higher forces $(9 \mathrm{pN})$ (Seo et al. 2016). A similar mechanism was used to mechanically deflect and activate inner ear hair cells by targeting MNPs to endogenous membrane glycoproteins. Cubic $\operatorname{MNPs}\left(\mathrm{Zn}_{0.4} \mathrm{Fe}_{2.6} \mathrm{O}_{4}\right)$ conjugated to concanavalin A were bound to glycoproteins on hair bundles and a pulsed magnetic field applied to induce movement of the hair bundle (Fig. 2). Magnetic field displacement of the hair bundle increased intracellular calcium, indicating activation of the hair cells (Lee et al. 2014). Magnetic activation of endogenous receptors has also been applied to enhance stem cell differentiation. MNPs functionalized with UM206, a synthetic ligand for 
S.A. Stanley and J.M. Friedman

A

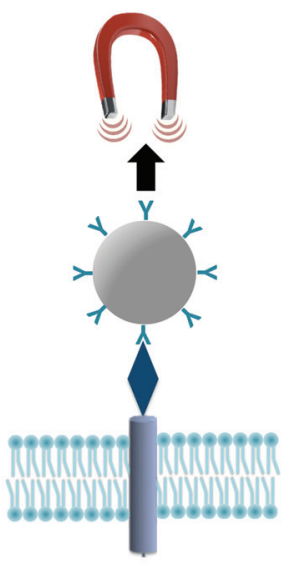

C

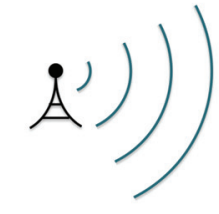

B

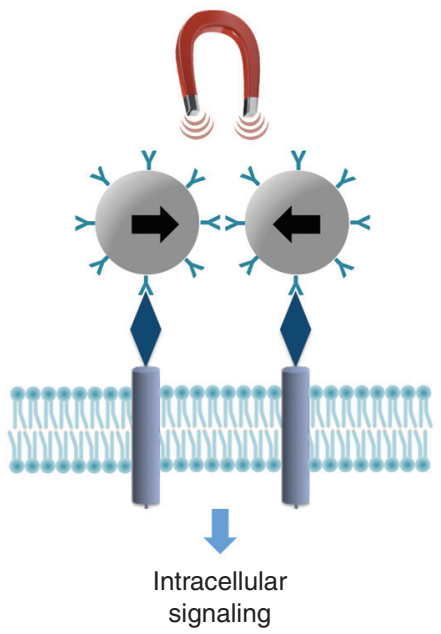

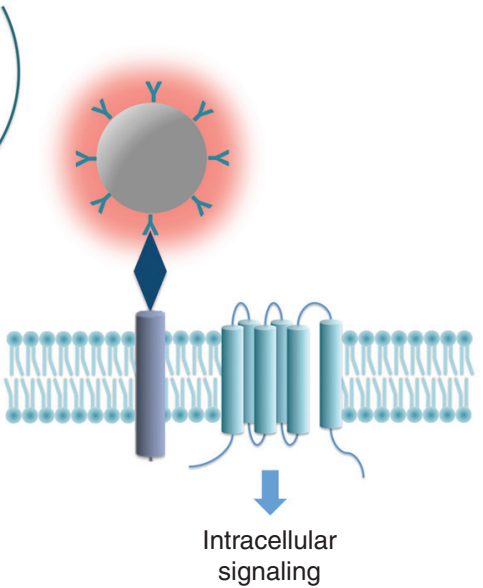

Figure 1. Schematic representation of mechanisms of magnet activation of cells using external nanoparticles. (A) Magnetic field treatment of cells decorated with magnetic nanoparticles (MNPs) functionalized to modified cell-surface proteins (e.g., Notch receptors) results in pulling on the cell membrane and mechanical activation of cells. (B) Magnetic field treatment of cells decorated with MNPs functionalized to cell-surface receptors (e.g., T-cell receptors, Death receptor 4) results in dipole-dipole interaction, oligomerization of the receptors, and downstream signaling. $(C)$ Oscillating magnetic field treatment induces nanoparticle heating of cells decorated with MNPs functionalized to cell-surface proteins or modified temperature-dependent ion channels (e.g., TRPV1). This results in activation of endogenous or transgenically expressed heat-sensitive ion channels and cell activation.

Wnt receptor, were used to mechanically activate the receptor (Rotherham et al. 2018) that, in turn, increased bone mineralization in chick femurs ex vivo. Magnetic field treatment of stem cells treated with MNPs targeting the mechanosensitive TREK-1 channel using anti-TREK Ab, or to the Arg-Gly-Asp (RGD)-binding sites of integrins also increased bone mineralization in vitro and ex vivo (Henstock et al. 2014).
Other studies have used MNPs to examine the role of mechanical force on neurons. Tay et al. (2016) incubated starch or chitosan-coated MNPs that were endocytosed by cultured cortical neurons and found that, in a magnetic field, a mechanical force in the piconewton range was exerted on the neurons and increased intracellular calcium. They also found that the signal increased with longer exposures to the magnetic 
Electromagnetic Regulation of Cell Activity

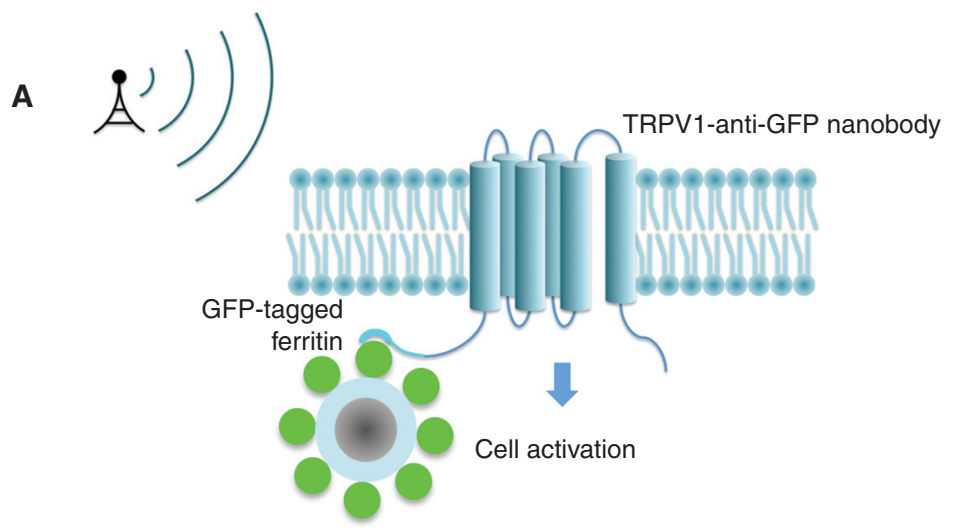

B

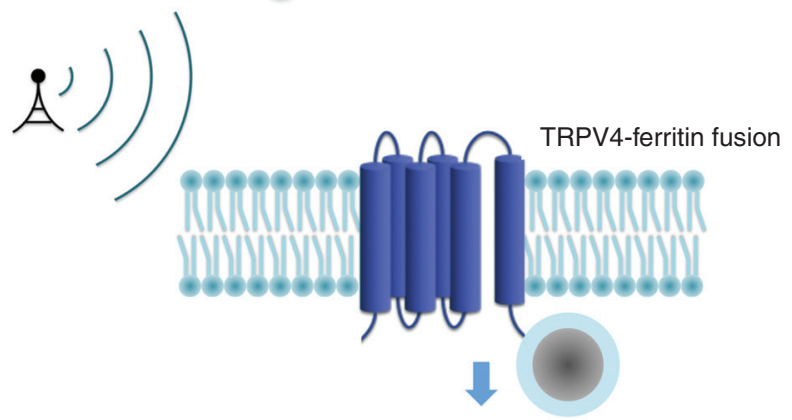

Cell activation

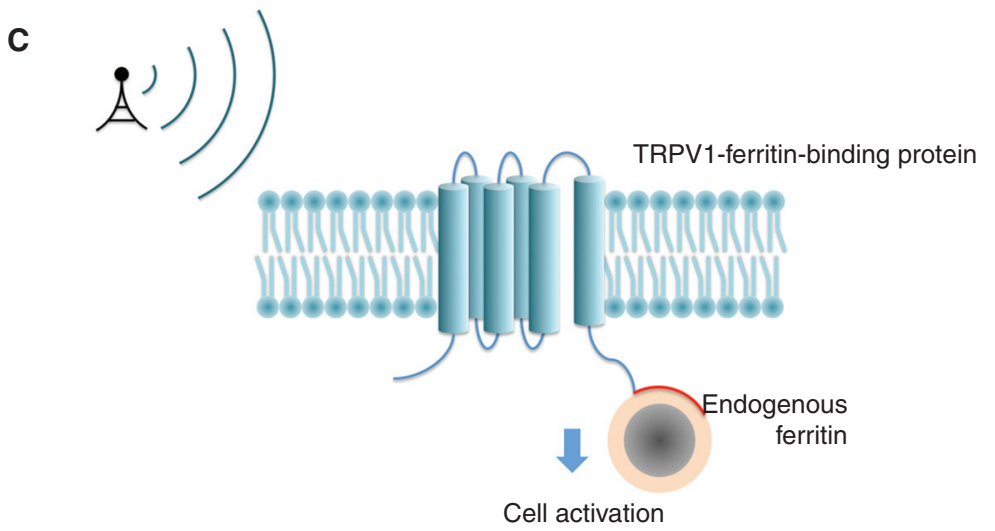

Figure 2. Schematic representation of mechanisms of magnet activation of cells using genetically encoded nanoparticles. (A) Target cells express a green fluorescent protein (GFP)-tagged ferritin chimeric peptide that self-assembles into a protein shell and generates iron oxide nanoparticles. GFP-ferritin tethers to TRPV1 ion channels modified with an amino-terminal fusion to a camelid anti-GFP nanobody. Energy from oscillating or gradient magnetic fields is absorbed by the nanoparticle, opening the TRPV1 channel and resulting in cell activation. (B) Target cells express TRPV4 ion channels with a carboxy-terminal fusion to a ferritin chimeric peptide. The ferritin self-assembles into a protein shell and generates iron oxide nanoparticles. Energy from oscillating or gradient magnetic fields is absorbed by the nanoparticle, opening the TRPV4 channel resulting in cell activation. (C) Target cells express TRPV1 (or TRPV4) modified by carboxy-terminal fusion to a ferritinbinding protein. This tethers endogenous ferritin and its iron oxide nanoparticle to the channel. Energy from oscillating or gradient magnetic fields is absorbed by the nanoparticle, opening the TRPV1 (or TRPV4) channel resulting in cell activation. 
field treatment and the calcium signal was propagated along neurites. This magnetically induced calcium signal was insensitive to temperature changes but blocked by $\omega$-conotoxin GVIA, an inhibitor of mechanosensitive Ntype calcium channels. MNPs have also been used to activate neurons and alter their growth. In these studies, the application of a magnetic field-initiated neurite outgrowth of chick embryo neurons decorated with MNPs functionalized with anti- $\beta$ integrin antibodies (Fass and Odde 2003). In the same study, Fass and Odde showed that the effects of the force were dependent on the rate that the increased force was applied. When the force was applied gradually, neurite outgrowth increased but rapid increases in force inhibited the neurons and reduced neurite outgrowth. Other studies have shown that application of a mechanical force in the low piconewton range to cultured neurons with internalized MNPs can lead to redistribution of the intracellular tau protein. Furthermore, as the applied force increased, the polarity of the tau protein was reorganized $(<70 \mathrm{pN}$ ) (Kunze et al. 2015), eventually leading to displacement of cells. Magnetic fields and MNPs have been used to manipulate organelles to modify cell activity. MNPs targeted to tropomyosin receptor kinase B (TrkB)-signaling endosomes were used to move the endosomes away from growth cones using an applied magnetic field in retinal ganglion cells and this was sufficient to stop neurite outgrowth (Steketee et al. 2011).

\section{Aggregation to Activate Cell Signaling}

Magnetic fields can also be used to aggregate MNPs to regulate cell activity. This relies on the fact that in the presence of a magnetic field, dipole-dipole interactions between the nanoparticles result in clustering. Thus, if the MNPs are tethered to cell-surface receptors, the magnetic field can induce the clustering or oligomerization of cell-surface receptors, in turn activating signaling. Several studies have applied this approach to regulate cell function. In immune cells, Perica et al. (2014) used iron-dextran MNPs coated with a chimeric major histocompatibility complex-IgG dimer and anti-CD28 antibody to stimulate $\mathrm{T}$-cell receptors. Incubating $\mathrm{T}$ cells with functionalized MNPs in the presence of a magnetic field significantly increased T-cell receptor aggregation. Application of a magnetic field to MNP-coated T cells transferred into mice with a subcutaneous B16 melanoma tumor, decreased tumor growth by eight- to tenfold and increased survival compared with control animals. Mannix et al. (2008) used a similar method for mast cell activation. Mast cells were preincubated with IgE to dinitrophenyl (DNP) and thus presented anti-DNP on their cell surface. When the primed mast cells were incubated with MNP functionalized with increasing amounts of DNP-Lys (1, 3, or 30 copies per particle), there was a dose-dependent increase in intracellular calcium. MNPs functionalized with just one DNP per particle bound to the mast cell surface but did not increase intracellular calcium. However, in the presence of a magnetic field, clustering of the cell-surface-bound MNPs resulted in receptor oligomerization and increased intracellular calcium. Treatment with a pulsatile magnetic field also produced an oscillating pattern of intracellular calcium.

Magnetic particles and applied magnetic fields have also been used to regulate apoptosis (Cho et al. 2012). Cancer cells express the DR4 death receptor at high levels and MNPs conjugated to anti-DR4 antibodies led to DR4 clustering that in turn activated caspase- 8 and caspase-3, leading to cell death in the presence of a magnetic field. The extent of apoptosis was proportional to magnetic field strength and treatment time but not field direction. This system was also tested in vivo in zebrafish by targeting MNPs to the ovarian tumor necrosis factor (TNF) receptor in single-cell embryos. Magnetic field treatment led to induction of apoptosis and morphological deformities in the zebrafish embryos that received ovarian TNF-receptortargeted MNPs but not in embryos without MNPs targeted to the ovarian TNF receptor.

\section{THERMAL EFFECTS OF EXTERNAL MAGNETIC PARTICLES}

Magnetic fields can also be used to generate a temperature increase adjacent to MNPs. MNPs 
with defined composition, size, and geometry will heat in an oscillating magnetic field. This local temperature change can then be transduced into a cell signal via effects on temperature-responsive proteins, in particular TRP ion channels. Initial studies targeted streptavidinfunctionalized MNPs to human embryonic kidney (HEK) cells coexpressing a biotinylated acceptor protein (at the cell surface) and the multimodal ion channel, transient receptor potential cation channel subfamily V, member 1, TRPV1. In these cells, a magnetic field induced increased intracellular calcium in vitro. Targeting the MNPs to temperature-sensing neurons in Caenorhabditis elegans also induced avoidance behavior via activation of an endogenous temperature-sensing pathway (Huang et al. 2010). Subsequent studies used an His-tagged TRPV1 channel and MNPs functionalized with antiHis. Exposure of HEK cells expressing Histagged TRPV1 and decorated with anti-His MNP to an oscillating magnetic field $(465 \mathrm{kHz})$ increased intracellular calcium and this was blocked by a TRP channel antagonist, ruthenium red. The increased calcium signal was then used to regulate a synthetic calcium-dependent response element upstream of an insulin complementary DNA (cDNA). Using this technique, magnetic fields were used to regulate calcium entry and calcium-dependent insulin gene expression in vivo after implantation of MNP-decorated cells. Exposure of the cells to a magnetic field, in turn, increased insulin production and release so lowered blood glucose in mice (Stanley et al. 2012).

Chen et al. (2015) applied a similar system to induce neural activation in mice. This system combined MNPs that were functionalized to prevent endocytosis with cells transduced with TRPV1 that were activated with an oscillating magnetic field. However, in this study, the MNPs were not directly targeted to TRPV1 but rather were injected in proximity to the TRPV1expressing neurons. Even when the MNPs were in proximity to the cells, an oscillating magnetic field-induced action potential in TRPV1expressing hippocampal neurons in vitro. In vivo, local central nervous system (CNS) injection of MNPs into the ventral tegmental area induced c-fos expression, a marker of neural activity, in cells transduced with TRPV1.

More recently, Munshi et al. (2017) have refined the use of MNPs for thermal activation of neurons. MNPs were functionalized by conjugation to neutravidin then attached to biotinylated A2B5 antibodies that bind neuronal glycosylated membrane proteins. Treatment of TRPV1-expressing hippocampal neurons significantly increased fluorescence of the calcium indicator, GCaMP6f, with $87 \%$ of cultured neurons responding within $5 \mathrm{sec}$. TRPV1 was transduced into the targeted brain regions in mice using adeno-associated virus or lentiviral delivery and the effects of oscillating magnetic field treatment after injection of the particles were then examined. The combination of TRPV1 expression and MNP delivery to the secondary motor cortex increased locomotion after exposure to a magnetic field. Unilateral TRPV1 expression and MNP injection into caudate putamen caused circling in mice with magnetic field treatment, whereas activation of the deeper striatum led to freezing behavior. These behaviors were rapid (within $15 \mathrm{sec}$ to $20 \mathrm{sec}$ ) and repeatable without any evidence of cell damage.

In aggregate, these studies show that external MNPs combined with static or oscillating magnetic fields have the ability to regulate cell activity through several mechanisms: mechanical forces, receptor activation by regulated oligomerization, or thermal effects. Functionalized MNPs can be directed to defined cell-surface markers (either endogenously expressed or genetically introduced) and to specific intracellular components allowing highly targeted modulation of function. Further work is needed to examine how long functionalized MNPs remain associated with their target in defined tissues but it is likely that repeated MNP administration will be needed for prolonged or repeated in vivo use. For some cell populations such as those in the CNS, the requirement for repeated intracranial injections could limit the application of these technologies. Thus, the use of external nanoparticles would be enhanced by the use of materials that were not degraded and could stably and durably induce cellular responses without the need for repeated injec- 
tions. An alternative approach to achieve durability of the response would be to use genetically encoded nanoparticles that are continually produced within the target cell thus obviating the need for repeated nanoparticle injection.

\section{GENETICALLY ENCODED PARTICLES}

Many different metals are components of enzymes and other biologic systems and several organisms are also capable of synthesizing complex structures from inorganic materials. Magnetotactic bacteria synthesize magnetite nanoparticles that align to the magnetic field (Uebe and Schuler 2016). Other organisms, such as earthworms, reduce toxic metals and the enzymes that catalyze these reactions can be exploited to generate luminescent nanoparticles (Sturzenbaum et al. 2013).

Although magnetite has been reported to be present in some mammalian tissues, this finding is somewhat controversial and the putative synthesis pathway remains unclear. However, almost all mammalian cells have intracellular stores of metals, in particular, iron, which is a key component of the respiratory chain in mitochondria. However, iron oxidation by the Fenton reaction can lead to extremely toxic metabolites and it is thus necessary to sequester iron. Among nearly all organisms other than yeast, iron is sequestered by ferritin, specialized proteins that form a protein shell around a core of different molecular forms of iron. In general, they sequester $\mathrm{Fe}(\mathrm{II})$, preventing conversion to $\mathrm{Fe}(\mathrm{III})$, which also produces free radicals. In the presence of iron, these proteins spontaneously form intracellular nanoparticles comprised primarily of iron oxides (Theil 2013).

The tertiary structure of ferritin is conserved across many species and is comprised of 24 protein subunits that self-assemble into dimers and then into a protein cage with an outer diameter of $\sim 12 \mathrm{~nm}$ and inner cavity of $7 \mathrm{~nm}$ to $8 \mathrm{~nm}$. The cavity is then filled with an iron oxide core, although other less well-characterized molecular forms of iron, including ferric iron, are also present in variable amounts. The protein shell in mammals is a heteromer of ferritin heavy chains (ferritin-H) and ferritin light chains (ferritin-L).
The heavy chain binds and oxidizes ferrous ions, while the light chain provides the nucleation site in the protein cage. The ratio of heavy- to lightchain ferritins varies between organisms and between tissues. The subunit proteins form a four-helix bundle with a left-handed twist and are arranged in 12 antiparallel pairs to make a rhombic dodecahedron structure. Iron likely enters the shell via channels at the threefold axis and the ferroxidase activity of the $\mathrm{H}$ chain catalyzes the oxidation of two $\mathrm{Fe}(\mathrm{II})$ ions with the intermediate production of $\mathrm{H}_{2} \mathrm{O}_{2}$. The light chain accelerates iron transport and may generate a more ordered iron oxide core (Bradley et al. 2014; Mehlenbacher et al. 2017).

The precise structure of native ferrihydrite core is variable depending on a variety of cellular conditions and thus remains unclear. The iron content of the ferritin core is variable but can be up to 4500 iron atoms. The core is typically described as hydrated ferric oxide $\left(5 \mathrm{Fe}_{2} \mathrm{O}_{3} \cdot 9 \mathrm{H}_{2} \mathrm{O}\right)$ (for a review, see Jutz et al. 2015). However, the magnetic properties, structure, and reactivity depend on the iron loading, size, and the ferritin protein shell. The most commonly studied ferritin, horse spleen ferritin, is $90 \%$ ferritin-L subunits. Studies examining the structure of the iron oxide particle in horse spleen ferritin as the number of iron atoms is gradually and controllably reduced suggest a polyphasic structure (Galvez et al. 2008). Using transmission electron microscopy (TEM), X-ray absorption near-edge spectroscopy (XANES), electron energy-loss spectroscopy (EELS), small-angle X-ray scattering (SAXS), and superconducting quantum interference device (SQUID) magnetic measurements, Galvez et al. concluded that there is a ferrihydrite core with magnetite shell. Because the ferrihydrite is more labile as the iron content decreases, the ferrihydrite core is removed and the magnetite shell remains and thus the particle size does not change. Other studies (Pan et al. 2009) also suggest a particle of constant diameter with variable iron density and central crystalline domains surrounded by more disordered material.

Although the magnetic properties of ferritin are likely to vary between species, tissues, and with iron loading, numerous studies have shown 
that cells that overexpress ferritin are affected by magnetic fields. Viral and transgenic overexpression of ferritin has been used as an MRI contrast agent in vitro and in vivo (Iordanova et al. 2010). Cells engineered to overexpress human ferritin heavy chain and divalent metal ion transferase 1 showed superparamagnetic properties allowing them to be separated from unmodified cells by a magnetic field (Kim et al. 2012). HEK cells overexpressing a ferritin chimeric peptide also move toward a magnetic field (Stanley et al. 2015). Finally, recent studies have used transfected or endogenous ferritin expression to target intracellularly generated iron nanoparticles to multimodal ion channels and shown a change in ion flux and cell activity when the engineered cells are exposed to a magnetic field.

The methods using genetically encoded nanoparticles for cell modulation generally have three components: (1) a signal in the form of a static or oscillating magnetic field; (2) iron nanoparticles surrounded by a shell of ferritin; and (3) a modified multimodal ion channel, TRPV1 or TRPV4. When a magnetic field is applied, energy is absorbed by iron within the ferritin shell and transferred to a tethered ion channel, which opens allowing ion entry into cells. Depending on the ionic selectivity of the channel, ion entry can activate or silence the targeted cells. This method has been validated in several papers some of which used a modified form of the multimodal cation channel, TRPV1. This channel is temperature responsive with a threshold of approximately $42^{\circ} \mathrm{C}$ but also responds to other stimuli such as $\mathrm{pH}$, chemical agonists (e.g., capsaicin), and possibly mechanical stimulation (Caterina et al. 1997). TRPV1 can be modified to interact with ferritin indirectly or directly. In one system (Stanley et al. 2015, 2016), TRPV1 was tethered to ferritin by fusion of the channel to an anti-green fluorescent protein (GFP) nanobody in combination with overexpression of a GFP-tagged ferritin chimera. The GFP ferritin construct co-expressed the heavy and light chains with a 1:1 ratio and cells expressing this light:heavy chain ratio have been shown to have increased iron loading (Iordanova et al. 2010). Oscillating or magnetic field treatment of HEK cells, stem cells, or neuronal cell lines expressing TRVP1 tethered to ferritin show increased intracellular calcium, phosphorylation of cyclic AMP response element-binding protein and cfos expression in a TRPV1-dependent manner. In patch clamp recordings, neurons expressing the anti-GFPTRPV1/GFP-ferritin constructs are depolarized and show an increased firing rate in ex vivo brain slices in a magnetic field. The field also induces an inward current in voltage clamp. Cells expressing a mutant form of TRPV1 engineered to gate chloride rather than calcium and sodium show an opposite effect on neural firing and outward rather than inward currents in response to a magnetic field. These studies confirm that the observed responses are TRPV1 dependent. These findings are consistent with those from a separate set of studies showing depolarization and activation of neurons expressing TRPV4 fused in frame to ferritin light and heavy chains (see below and Wheeler et al. 2016).

In vivo studies have confirmed the ability of ferritin tethered to TRPV1 to activate neurons. Oscillating or gradient magnetic field treatment of mice with adenoviral expression of anti-GFP TRPV1/GFP-ferritin in glucose-sensing neurons of the ventromedial hypothalamus increased blood glucose with a similar time course and to a similar extent to optogenetic stimulation of the same neural population. The increased glucose was a result of elevated glucagon secretion and a reduction of insulin secretion. Gradient magnetic field treatment of ventromedial hypothalamus glucose-sensing neurons expressing anti-GFP-TRPV1/GFP-ferritin also increased food intake in freely moving mice to a similar extent as optogenetic stimulation of these neurons. These studies also showed the glucose response was proportional to the strength of the applied magnetic field so this technique can be used to generate a graded response.

The TRPV4-ferritin fusion construct was also tested in vivo (Wheeler et al. 2016). The activity of this channel is also temperature dependent, with a threshold of $33^{\circ} \mathrm{C}$ (Guler et al. 2002) but the channel also responds to osmotic stimuli and agonist treatment. In this system, the ferritin chimeric peptide is directly fused 
to the carboxy-terminal of a truncated version of TRPV4 (TRPV4 $\left.4_{\triangle 760-871}\right)$, a construct named Magneto 2.0. Magnet treatment of HEK cells expressing Magneto 2.0 increased intracellular calcium in a TRPV4-dependent manner. Magnet treatment of excitatory neurons expressing Magneto 2.0 in the entorhinal cortex increased firing rate in ex vivo brain slices. In vivo, Magneto 2.0 was first expressed in the sensory neurons of zebrafish. Magnet treatment of these neurons increased intracellular calcium and induced coiling behavior, which was absent in wild-type fish or when TRPV4 was not fused to ferritin. Magneto 2.0 was also used to regulate cell activity in mice. When the construct was expressed in striatal dopamine receptor 1-expressing neurons, neurons that are known to be involved in reward behavior, mice showed greater preference for the region of a chamber treated with a magnetic field. The ability of the Magneto 2.0 construct to regulate cell activity in vitro has also been confirmed by other investigators (Duret et al. 2017).

Magnetic gating of ion channels has also been shown by inserting a ferritin-binding motif into TRP channels, which then tethers the channel to endogenous ferritin (Hutson et al. 2017). Hutson et al. showed that temperature-induced activation of TRPV1 or TRPV4 in chick embryos resulted in cardiac and craniofacial defects. They then used remote magnetic activation of TRPV channels to replicate the effect of elevated temperature. To do so, they generated carboxyterminal fusions of TRPV1 or TRPV4 to the ferritin-binding domain 5 (D5) of kininogen-1 to bind endogenously expressed ferritin (TRPV $1^{\text {FeRIC }}$ or TRPV4 ${ }^{\text {FeRIC }}$, respectively). Oscillating magnetic field treatment $(175 \mathrm{MHz}$, $36 \mathrm{uT}$ ) of HEK cells expressing these constructs increased fluorescence of the calcium indicator; GCaMP6 and the calcium changes were blunted by TRPV1 or V4 antagonists or with CRISPR/ Cas9 deletion of endogenous ferritin heavy chain. When TRPV $1^{\text {FeRIC }}$ or TRPV4 ${ }^{\text {FeRIC }}$ were electroporated into chick neural crest cells and then treated with an oscillating magnetic field, they observed an increase in structural heart and craniofacial defects, phenocopying the effects of temperature activation of these cells.
TRPV1 tethered to ferritin has also been used to regulate cell activity and transgene expression in a peripheral tissue (Stanley et al. 2015). Expression of anti-GFP-TRPV1/GFPferritin was combined with expression of a bioengineered insulin gene under the control of a calcium-dependent promoter in HEK cells. Oscillating or intermittent magnetic field treatment increased intracellular calcium and the release of proinsulin. When the constructs were expressed in the livers of diabetic mice, oscillating or intermittent magnetic field treatment of these mice increased plasma insulin and reduced blood glucose. In another demonstration of cell regulation in peripheral tissues, the anti-GFP-TRPV1/GFP-ferritin system was also used for magnetic field-regulated cell migration. Mosabbir and Truong (2018) showed a magnetic field-dependent increase in intracellular calcium in a stable HEK cell line expressing antiGFP-TRPV1/GFP-ferritin and the red calcium indicator, RCaMP. Next, they combined stable expression of anti-GFP-TRPV1/GFP-ferritin with expression of calcium-dependent Rho-A in HEK cells to regulate cell migration. Magnetic field treatment of these stably expressing cells increased cell protrusions. In addition, magnetic field treatment of cells stably expressing antiGFP-TRPV1/GFP-ferritin with a calciumdependent Rho-A promoted wound healing in an in vitro assay. The direction of cell growth could also be controlled by the magnetic field.

The system for magnetic control of cells expressing ion channel-ferritin complexes can also be used to inhibit cell activity. Mutation of an amino acid in the pore region of TRPM2 and TRPM8 (Kuhn et al. 2007) altered the ionic selectivity of these channels from cations to anions and an equivalent mutation in TRPV1 (TRPV1 ${ }^{\text {Mutant }}$ ) also changed the ionic selectivity to anions (Stanley et al. 2016). HEK or neural cells expressing anti-GFP-TRPV1 ${ }^{\text {Mutant }} / \mathrm{GFP}$ ferritin show increased fluorescence of a chloride indicator, MQAE, and as described above, in ex vivo brain slices, electrophysiological recordings from magnet-treated cells expressing anti-GFP-TRPV $1^{\text {Mutant } / G F P-f e r r i t i n ~ s h o w ~ h y-~}$ perpolarization and silencing of neural activity in response to a magnetic field. This construct 
was then introduced into the glucose-sensing neurons of the ventromedial hypothalamus in mice and magnet treatment of these mice decreased blood glucose and suppressed feeding, effects that were the opposite of those seen when the same neural population was activated.

The mechanisms by which magnetic fields interact with ion channel-ferritin constructs to regulate cell activity remain unclear. Theoretical calculations (Meister 2016) suggest that static or oscillating magnetic fields acting on ferritin would not generate sufficient mechanical force or heat to open the associated channel via classical mechanisms. However, recent theoretical work supported by experimental findings suggest an alternative mechanism based on the magnetocaloric effect (Duret et al. 2017). Duret et al. propose that in the absence of a magnetic field, the magnetic moments in ferritin are orientated in a random fashion and therefore have high magnetic entropy. However, in the presence of a magnetic field, the moments align with the field and reduce magnetic entropy. To compensate for the loss of magnetic entropy, molecular vibrations increase, resulting in heat production. Duret et al. calculated that the temperature change would be sufficient to increase the probability of TRPV1 or TRPV4 channel opening and would open approximately one in ten expressed channels. Because the conductance of TRP channels is relatively high (TRPV1 95-103 picosiemens [Cheng et al. 2012], TRPV4 60 picosiemens [Strotmann et al. 2000]) compared with channelrhoposin (50 femtosiemens [Nagel et al. 2003]), opening this proportion of channels in a neuron or HEK cell would lead to detectable calcium influx and depolarization. Supporting their theoretical calculations, Duret et al. (2017) show that the calcium response to magnetic field treatment is lost in cells expressing a temperature-insensitive TRPV4-ferritin construct. Similarly, Hutson et al. (2017) show that the calcium response to oscillating magnetic field treatment is also lost in cells expressing a temperature-insensitive TRPV1 fused to a ferritin-binding domain. Although this mechanism is plausible, additional magnetomechanical and magnetothermal mechanisms of ion channel activation by iron- loaded ferritin are under consideration (M Barbic, in prep.). Those include the cluster-paramagnetic magnetocaloric effect in iron-loaded ferritin, diamagnetic strain on the TRP channels caused by the magnetic fields and magnetic field gradients from the iron-loaded ferritin, the effect of rapidly fluctuating magnetic fields from the iron-loaded ferritin on the TRP channels, and additional thermal relaxation mechanisms in iron-loaded ferritin that has previously not been considered, such as the Einstein-de Haas thermal relaxation mode (M Barbic, in prep.). Many of such proposed magnetic-based physical mechanisms that might influence TRP ion channels will remain challenging to theoretically evaluate until the proper chemical, structural, and magnetic spin structure analysis of iron within the ferritin protein is properly performed experimentally on a single-particle-protein level (M Barbic, pers. comm.).

\section{CLINICAL IMPLICATIONS AND FUTURE CONSIDERATIONS}

The electromagnetic regulation of cellular activity in vivo has numerous potential clinical applications in the field of bioelectronic medicine. There are also some challenges that would need to be overcome, especially for modulation of the activity of deeper structures in the CNS. Currently, neural stimulation by deep brain stimulation is used for many clinical indications and in a range of anatomical sites, from peripheral nerves to the CNS. Nerve stimulation is often invasive, requiring a permanent implant that can become infected or sclerose and so lose activity over time. These procedures often require special expertise and sophisticated equipment, which restricts patient access to a limited number of centers. Finally, the invasive nature means most implants are limited to a single site or adjacent regions. Noninvasive regulation of neural activity using electromagnetic fields could, in theory, allow modulation of multiple sites in a circuit without the need for implants.

A principal obstacle to the implementation of this method in clinical settings will be the delivery of the constructs into cells and the subsequent generation of a sufficiently strong 
magnetic field locally to activate cells. Many of the other components, however, are already in clinical usage. Iron oxide nanoparticles have been approved by the Food and Drug Administration (FDA) as treatments for iron deficiency in select renal failure patients (Provenzano et al. 2009). The use of functionalized particles targeted to neuronal membrane proteins may allow anatomically targeted cell regulation in clinical settings without the need to express additional tags or proteins in target cells. However, as mentioned, the durability of these external particles has not been determined in clinical studies and further work would be needed to determine how long these exogenous particles remain bound to the cell membrane.

The alternative approach using modified ion channels and genetically encoded nanoparticles would not need repeated nanoparticle administration but would need to use gene therapy to deliver a neuromodulatory construct in vivo. However, gene delivery into cells ex vivo is already in common usage in Car-T cells and magnetic modulation of cell activity of the reintroduced cells is possible. Ion flux has been shown to modulate immune cell function (Ohhora and Rao 2008; Cahalan and Chandy 2009), and, in principle, magnetic gating of channels could be used to either activate immune cells or reduce their activity such as to quell cytokine storms. However, further studies are needed to confirm the effects of magnetic gating of channels on immune cell function. In addition, means for delivering a sufficiently strong field to gate the channel in vivo would need to be developed.

As mentioned, the application of magnetogenetic technologies for neurologic disorders would require that the channel and ferritin constructs (or a ferritin-binding motif) be delivered to neurons in vivo. A number of gene therapies have now been approved in Europe (Touchot and Flume 2017) and the United States (Dias et al. 2018) and many clinical trials (from phase I to phase III) are underway to test the safety and efficacy of other therapies (Ginn et al. 2013). Most of these approaches use viral vectors such as adeno-associated virus. Recent advances in adeno-associated virus technology could allow CNS transduction following peripheral in- jection (Chan et al. 2017) and offer the promise of cell-type-specific targeting using specific viral capsid proteins (Buning et al. 2015) or cell-typespecific promoters (Kim et al. 2015).

Approaches using exogenous or endogenous particles will require devices to generate electromagnetic fields, typically at millitesla field strengths. This field strength is orders of magnitude higher than those sensed by birds, which are in the microtesla range (Wiltschko and Wiltschko 2012). A range of devices would likely be needed, depending on whether these will be patient or clinician administered, as well as the anatomical site and the duration of treatment that is required. For example, devices for superficial sites, such as modulation of peripheral nerves to treat pain, would need to be lightweight, rechargeable, and easy to use. These devices could be readily developed with existing technology or perhaps even employ strong handheld magnets. Therefore, applications targeting peripheral nerves or autonomic ganglia, for example, to treat pain or regulate bladder function, are likely to be feasible and offer the possibility of rapid, regulated control of nerve function. However, generating sufficient electromagnetic fields for deep structures, such as the subthalamic nucleus currently targeted in Parkinson's disease by deep brain stimulation (Antonini et al. 2018), is more challenging. Larger power sources (likely $>1000 \mathrm{~W}$ ) are needed to generate millitesla magnetic fields at this depth and, while these are feasible for fixed devices, they are not yet possible for wearable devices. Instruments capable of delivering strong magnetic fields are already approved and in clinical usage for the treatment of depression using TMS. TMS can deliver a brief, focal magnetic field of $1.5 \mathrm{~T}$ in superficial brain regions (Perera et al. 2016) but TMS devices are large, fixed, and are suitable only for intermittent use. However, TMS devices require significantly higher power than a magnetic stimulation device would need as TMS needs to deliver higher peak voltages and currents to generate a short duration pulse. Thus, technological innovations in both construct delivery and electromagnetic devices will be necessary to develop clinical applications for electromagnetic cell regulation, 
especially for structures that are deeper in the brain.

Finally, the ability to modulate neural activity in deeper brain regions will likely require that more sensitive magnetogenetic methods, modulated at a lower field strength, be developed. Although theoretical analyses and experimental data support a magnetocaloric mechanism, the precise means by which energy is transduced from ferritin to the channel is still under investigation. A fuller understanding of the operative mechanism might thus enable the development of more efficient systems that require lower field strengths. Indeed, it is possible that more than one mechanism contributes in an additive or some other manner. Thus, a theoretical explanation of the empirical data from several different groups may provide insights into a heretofore unexplored mechanism. Richard Feynman stated "Progress in science comes when experiments contradict theory." Thus, in addition to its practical implications, the elucidation of the mechanism by which ferritin can gate ion channels in a magnetic field may also provide new vistas into the physical world.

\section{CONCLUDING REMARKS}

Electromagnetic regulation of cell activity has been used for many decades to investigate physiological functions in vitro and in vivo. There are many advantages to using electromagnetic signals to regulate cell function. The signal can be applied remotely (even in vivo), switched on and off rapidly, quickly titrated to produce a graded response, and can modulate cell function over a wide range of scales from a fraction of a cell to whole animals. For many studies, strong rare earth magnets or simple electromagnets are sufficient to control cell activity. In addition, with defined nanoparticle composition and functionalization, these tools can target specific cellular proteins and generate a variety of stimuli from thermal effects to activation of intracellular signaling pathways. The size, composition, and target of external nanoparticles can be carefully tailored to the required application but these may need to be given repeatedly to investigate biological processes in vivo. Recent studies have shown that genetically encoded nanoparticles can effectively modulate cell activity. As we learn more about the chemical composition and crystal structure of iron oxide nanoparticles generated in ferritin and how these interact with magnetic fields, it should be possible to engineer a wider range and better tools for cell regulation. A number of studies have already used directed evolution to generate mutant ferritins, producing nanoparticles that confer increased cellular magnetization (Matsumoto et al. 2015; Liu et al. 2016). Similarly, modifications to the multimodal channels binding ferritin and testing additional ion channels should extend the available tools for cell modulation. In combination with technologies for tracing neural circuits and in vivo imaging, electromagnetic tools to remotely regulate cell activity allow us to investigate the physiological roles of specific cells and pathways in health and disease. With time, these technologies may form the basis for novel forms of bioelectronic medical therapies.

\section{ACKNOWLEDGMENTS}

Support for this work was provided by the $\mathrm{Na}$ tional Institutes of Health (NIH) (MH105941 and 1R01NS097184), the American Diabetes Association (ADA \#1-17-ACE-31), EinsteinMt. Sinai Diabetes Research Center Pilot and Feasibility Award (P30DK020541), and the Alexander and Alexandrine Sinsheimer Scholar Award.

\section{REFERENCES}

Alexander GM, Rogan SC, Abbas AI, Armbruster BN, Pei Y, Allen JA, Nonneman RJ, Hartmann J, Moy SS, Nicolelis MA, et al. 2009. Remote control of neuronal activity in transgenic mice expressing evolved $G$ protein-coupled receptors. Neuron 63: 27-39.

Antonini A, Moro E, Godeiro C, Reichmann H. 2018. Medical and surgical management of advanced Parkinson's disease. Mov Disord doi: 10.1002/mds.27349.

Arenkiel BR, Klein ME, Davison IG, Katz LC, Ehlers MD. 2008. Genetic control of neuronal activity in mice conditionally expressing TRPV1. Nat Methods 5: 299-302.

Berndt A, Yizhar O, Gunaydin LA, Hegemann P, Deisseroth K. 2009. Bi-stable neural state switches. Nat Neurosci 12: 229-234. 
Boyden ES, Zhang F, Bamberg E, Nagel G, Deisseroth K. 2005. Millisecond-timescale, genetically targeted optical control of neural activity. Nat Neurosci 8: 1263-1268.

Bradley JM, Moore GR, Le Brun NE. 2014. Mechanisms of iron mineralization in ferritins: One size does not fit all. J Biol Inorg Chem 19: 775-785.

Buning H, Huber A, Zhang L, Meumann N, Hacker U. 2015. Engineering the AAV capsid to optimize vector-host-interactions. Curr Opin Pharmacol 24: 94-104.

Cahalan MD, Chandy KG. 2009. The functional network of ion channels in T lymphocytes. Immunol Rev 231: 59-87.

Caterina MJ, Schumacher MA, Tominaga M, Rosen TA, Levine JD, Julius D. 1997. The capsaicin receptor: A heat-activated ion channel in the pain pathway. Nature 389: 816-824.

Chan KY, Jang MJ, Yoo BB, Greenbaum A, Ravi N, Wu WL, Sanchez-Guardado L, Lois C, Mazmanian SK, Deverman $\mathrm{BE}$, et al. 2017. Engineered AAVs for efficient noninvasive gene delivery to the central and peripheral nervous systems. Nat Neurosci 20: 1172-1179.

Chen R, Romero G, Christiansen MG, Mohr A, Anikeeva P. 2015. Wireless magnetothermal deep brain stimulation. Science 347: 1477-1480.

Cheng W, Yang F, Liu S, Colton CK, Wang C, Cui Y, Cao X, Zhu MX, Sun C, Wang K, et al. 2012. Heteromeric heatsensitive transient receptor potential channels exhibit distinct temperature and chemical response. J Biol Chem 287: 7279-7288

Cho MH, Lee EJ, Son M, Lee JH, Yoo D, Kim JW, Park SW, Shin JS, Cheon J. 2012. A magnetic switch for the control of cell death signalling in in vitro and in vivo systems. Nat Mater 11: 1038-1043.

Crick FHS, Hughes AFW. 1950. The physical properties of cytoplasm: A study by means of the magnetic particle method. Part I: Experimental. Exp Cell Res 1: 37-80.

Dagnew R, Lin YY, Agatep J, Cheng M, Jann A, Quach V, Monroe M, Singh G, Minasyan A, Hakimian J, et al. 2017. CerebraLux: A low-cost, open-source, wireless probe for optogenetic stimulation. Neurophotonics 4: 045001.

Dias MF, Joo K, Kemp JA, Fialho SL, da Silva Cunha A Jr, Woo SJ, Kwon YJ. 2018. Molecular genetics and emerging therapies for retinitis pigmentosa: Basic research and clinical perspectives. Prog Retin Eye Res 63: 107-131.

Duret G, Polali S, Anderson ED, Bell AM, Tzouanas CN, Avants BW, Robinson JT. 2017. Magnetic entropy as a gating mechanism for magnetogenetic ion channels. bioRxiv doi: 10.1101/148379.

Fass JN, Odde DJ. 2003. Tensile force-dependent neurite elicitation via anti- $\beta 1$ integrin antibody-coated magnetic beads. Biophys J 85: 623-636.

Frazier SJ, Cohen BN, Lester HA. 2013. An engineered glutamate-gated chloride ( $\mathrm{GluCl}$ ) channel for sensitive, consistent neuronal silencing by ivermectin. J Biol Chem 288: 21029-21042.

Freake MJ, Muheim R, Phillips JB. 2006. Magnetic maps in animals: A theory comes of age? Q Rev Biol 81: 327-347.

Galvez N, Fernandez B, Sanchez P, Cuesta R, Ceolin M, Clemente-Leon M, Trasobares S, Lopez-Haro M, Calvino JJ, Stephan O, et al. 2008. Comparative structural and chemical studies of ferritin cores with gradual removal of their iron contents. J Am Chem Soc 130: 8062-8068.
Ginn SL, Alexander IE, Edelstein ML, Abedi MR, Wixon J. 2013. Gene therapy clinical trials worldwide to 2012-An update. J Gene Med 15: 65-77.

Guler AD, Lee H, Iida T, Shimizu I, Tominaga M, Caterina M. 2002. Heat-evoked activation of the ion channel, TRPV4. J Neurosci 22: 6408-6414.

Halperin D, Clark SS, Fu K, Heydt-Benjamin TS, Defend B, Kohno T, Ransford B, Morgan W, Maisel WH. 2008. Pacemakers and implantable cardiac defibrillators: Software radio attacks and zero-power defenses. IEEE Symposium on Security and Privacy, pp. 129-142. Oakland, CA.

Henstock JR, Rotherham M, Rashidi H, Shakesheff KM, El Haj AJ. 2014. Remotely activated mechanotransduction via magnetic nanoparticles promotes mineralization synergistically with bone morphogenetic protein 2: Applications for injectable cell therapy. Stem Cells Transl Med 3: 1363-1374.

Huang H, Delikanli S, Zeng H, Ferkey DM, Pralle A. 2010. Remote control of ion channels and neurons through magnetic-field heating of nanoparticles. Nat Nanotechnol 5: 602-606.

Hutson MR, Keyte AL, Hernandez-Morales M, Gibbs E, Kupchinsky ZA, Argyridis I, Erwin KN, Pegram K, Kneifel M, Rosenberg PB, et al. 2017. Temperature-activated ion channels in neural crest cells confer maternal feverassociated birth defects. Sci Signal 10: eaa14055.

Iordanova B, Robison CS, Ahrens ET. 2010. Design and characterization of a chimeric ferritin with enhanced iron loading and transverse NMR relaxation rate. J Biol Inorg Chem 15: 957-965.

Jutz G, van Rijn P, Santos Miranda B, Boker A. 2015. Ferritin: A versatile building block for bionanotechnology. Chem Rev 115: 1653-1701.

Kedzior KK, Gellersen HM, Brachetti AK, Berlim MT. 2015. Deep transcranial magnetic stimulation (DTMS) in the treatment of major depression: An exploratory systematic review and meta-analysis. J Affect Disord 187: 73-83.

Kim T, Moore D, Fussenegger M. 2012. Genetically programmed superparamagnetic behavior of mammalian cells. J Biotechnol 162: 237-245.

Kim Y, Kim T, Rhee JK, Lee D, Tanaka-Yamamoto K, Yamamoto Y. 2015. Selective transgene expression in cerebellar Purkinje cells and granule cells using adenoassociated viruses together with specific promoters. Brain Res 1620: 1-16.

Kim CK, Adhikari A, Deisseroth K. 2017. Integration of optogenetics with complementary methodologies in systems neuroscience. Nat Rev Neurosci 18: 222-235.

Kuhn FJ, Knop G, Luckhoff A. 2007. The transmembrane segment $\mathrm{S} 6$ determines cation versus anion selectivity of TRPM2 and TRPM8. J Biol Chem 282: 27598-27609.

Kunze A, Tseng P, Godzich C, Murray C, Caputo A, Schweizer FE, Di Carlo D. 2015. Engineering cortical neuron polarity with nanomagnets on a chip. ACS Nano 9: $3664-3676$

Lee JH, Kim JW, Levy M, Kao A, Noh SH, Bozovic D, Cheon J. 2014. Magnetic nanoparticles for ultrafast mechanical control of inner ear hair cells. ACS Nano 8: 6590-6598.

Lerchner W, Xiao C, Nashmi R, Slimko EM, van TL, Lester HA, Anderson DJ. 2007. Reversible silencing of neuronal 
excitability in behaving mice by a genetically targeted ivermectin-gated $\mathrm{Cl}^{-}$channel. Neuron 54: 35-49.

Liu X, Lopez PA, Giessen TW, Giles M, Way JC, Silver PA. 2016. Engineering genetically-encoded mineralization and magnetism via directed evolution. Sci Rep 6: 38019 .

Magnus CJ, Lee PH, Atasoy D, Su HH, Looger LL, Sternson SM. 2011. Chemical and genetic engineering of selective ion channel-ligand interactions. Science 333: 1292-1296.

Mannix RJ, Kumar S, Cassiola F, Montoya-Zavala M, Feinstein E, Prentiss M, Ingber DE. 2008. Nanomagnetic actuation of receptor-mediated signal transduction. Nat Nanotechnol 3: 36-40.

Matsumoto Y, Chen R, Anikeeva P, Jasanoff A. 2015. Engineering intracellular biomineralization and biosensing by a magnetic protein. Nat Commun 6: 8721 .

Mehlenbacher M, Poli M, Arosio P, Santambrogio P, Levi S, Chasteen ND, Bou-Abdallah F. 2017. Iron oxidation and core formation in recombinant heteropolymeric human ferritins. Biochemistry 56: 3900-3912.

Meister M. 2016. Physical limits to magnetogenetics. eLife 5: e17210.

Melo CA, Lima AL, Brasil IR, Castro e Silva O Jr, Magalhaes DV, Marcassa LG, Bagnato VS. 2001. Characterization of light penetration in rat tissues. J Clin Laser Med Surg 19: 175-179.

Mosabbir AA, Truong K. 2018. Genetically encoded circuit for remote regulation of cell migration by magnetic fields. ACS Synth Biol 7: 718-726.

Munshi R, Qadri SM, Zhang Q, Castellanos Rubio I, Del Pino P, Pralle A. 2017. Magnetothermal genetic deep brain stimulation of motor behaviors in awake, freely moving mice. eLife 6: 27069.

Nagel G, Szellas T, Huhn W, Kateriya S, Adeishvili N, Berthold P, Ollig D, Hegemann P, Bamberg E. 2003. Channelrhodopsin-2, a directly light-gated cation-selective membrane channel. Proc Natl Acad Sci 100: 13940 13945.

Nawaratne V, Leach K, Suratman N, Loiacono RE, Felder CC, Armbruster BN, Roth BL, Sexton PM, Christopoulos A. 2008. New insights into the function of M4 muscarinic acetylcholine receptors gained using a novel allosteric modulator and a DREADD (designer receptor exclusively activated by a designer drug). Mol Pharmacol 74: 11191131.

Oh-hora M, Rao A. 2008. Calcium signaling in lymphocytes. Curr Opin Immunol 20: 250-258.

Packer AM, Peterka DS, Hirtz JJ, Prakash R, Deisseroth K, Yuste R. 2012. Two-photon optogenetics of dendritic spines and neural circuits. Nat Methods 9: 1202-1205.

Pan YH, Sader K, Powell JJ, Bleloch A, Gass M, Trinick J, Warley A, Li A, Brydson R, Brown A. 2009. 3D morphology of the human hepatic ferritin mineral core: New evidence for a subunit structure revealed by single particle analysis of HAADF-STEM images. J Struct Biol 166: 22 31.

Perera T, George MS, Grammer G, Janicak PG, PascualLeone A, Wirecki TS. 2016. The Clinical TMS Society consensus review and treatment recommendations for TMS therapy for major depressive disorder. Brain Stimul 9: 336-346.
Perica K, Tu A, Richter A, Bieler JG, Edidin M, Schneck JP. 2014. Magnetic field-induced T cell receptor clustering by nanoparticles enhances $\mathrm{T}$ cell activation and stimulates antitumor activity. ACS Nano 8: 2252-2260.

Petreanu L, Huber D, Sobczyk A, Svoboda K. 2007. Channelrhodopsin-2-assisted circuit mapping of long-range callosal projections. Nat Neurosci 10: 663-668.

Provenzano R, Schiller B, Rao M, Coyne D, Brenner L, Pereira BJ. 2009. Ferumoxytol as an intravenous iron replacement therapy in hemodialysis patients. Clin J Am Soc Nephrol 4: 386-393.

Rotherham M, Henstock JR, Qutachi O, El Haj AJ. 2018. Remote regulation of magnetic particle targeted Wnt signaling for bone tissue engineering. Nanomedicine 14: 173-184.

Seo D, Southard KM, Kim JW, Lee HJ, Farlow J, Lee JU, Litt DB, Haas T, Alivisatos AP, Cheon J, et al. 2016. A mechanogenetic toolkit for interrogating cell signaling in space and time. Cell 165: 1507-1518.

Setsompop K, Feinberg DA, Polimeni JR. 2016. Rapid brain MRI acquisition techniques at ultra-high fields. NMR Biomed 29: 1198-1221.

Stanley SA, Gagner JE, Damanpour S, Yoshida M, Dordick JS, Friedman JM. 2012. Radio-wave heating of iron oxide nanoparticles can regulate plasma glucose in mice. Science 336: 604-608.

Stanley SA, Sauer J, Kane RS, Dordick JS, Friedman JM. 2015. Remote regulation of glucose homeostasis in mice using genetically encoded nanoparticles. Nat Med 21: 92-98.

Stanley SA, Kelly L, Latcha KN, Schmidt SF, Yu X, Nectow AR, Sauer J, Dyke JP, Dordick JS, Friedman JM. 2016. Bidirectional electromagnetic control of the hypothalamus regulates feeding and metabolism. Nature 531: $647-650$.

Stauffer PR, Cetas TC, Jones RC. 1984. Magnetic induction heating of ferromagnetic implants for inducing localized hyperthermia in deep-seated tumors. IEEE Trans Biomed Eng 31: 235-251.

Steketee MB, Moysidis SN, Jin XL, Weinstein JE, PitaThomas W, Raju HB, Iqbal S, Goldberg JL. 2011. Nanoparticle-mediated signaling endosome localization regulates growth cone motility and neurite growth. Proc Natl Acad Sci 108: 19042-19047.

Sternson SM, Roth BL. 2014. Chemogenetic tools to interrogate brain functions. Annu Rev Neurosci 37: 387-407.

Strotmann R, Harteneck C, Nunnenmacher K, Schultz G, Plant TD. 2000. OTRPC4, a nonselective cation channel that confers sensitivity to extracellular osmolarity. Nat Cell Biol 2: 695-702.

Sturzenbaum SR, Hockner M, Panneerselvam A, Levitt J, Bouillard JS, Taniguchi S, Dailey LA, Ahmad Khanbeigi R, Rosca EV, Thanou M, et al. 2013. Biosynthesis of luminescent quantum dots in an earthworm. Nat Nanotechnol 8: 57-60.

Tay A, Kunze A, Murray C, Di Carlo D. 2016. Induction of calcium influx in cortical neural networks by nanomagnetic forces. ACS Nano 10: 2331-2341.

Theil EC. 2013. Ferritin: The protein nanocage and iron biomineral in health and in disease. Inorg Chem 52: 12223-12233. 
S.A. Stanley and J.M. Friedman

Touchot N, Flume M. 2017. Early insights from commercialization of gene therapies in Europe. Genes (Basel) 8: E78.

Uebe R, Schuler D. 2016. Magnetosome biogenesis in magnetotactic bacteria. Nat Rev Microbiol 14: 621-637.

Wang H, Peca J, Matsuzaki M, Matsuzaki K, Noguchi J, Qiu L, Wang D, Zhang F, Boyden E, Deisseroth K, et al. 2007. High-speed mapping of synaptic connectivity using photostimulation in channelrhodopsin-2 transgenic mice. Proc Natl Acad Sci 104: 8143-8148.

Wheeler MA, Smith CJ, Ottolini M, Barker BS, Purohit AM, Grippo RM, Gaykema RP, Spano AJ, Beenhakker MP,
Kucenas S, et al. 2016. Genetically targeted magnetic control of the nervous system. Nat Neurosci 19: 756-761.

Wiltschko R, Wiltschko W. 2012. Magnetoreception. Adv Exp Med Biol 739: 126-141.

Zemelman BV, Nesnas N, Lee GA, Miesenbock G. 2003 Photochemical gating of heterologous ion channels: Remote control over genetically designated populations of neurons. Proc Natl Acad Sci 100: 1352-1357.

Zhang F, Wang LP, Brauner M, Liewald JF, Kay K, Watzke N Wood PG, Bamberg E, Nagel G, Gottschalk A, et al. 2007. Multimodal fast optical interrogation of neural circuitry. Nature 446: 633-639. 


\section{$\&_{\mathrm{CSH}}^{\infty} \&$ Cold Spring Harbor

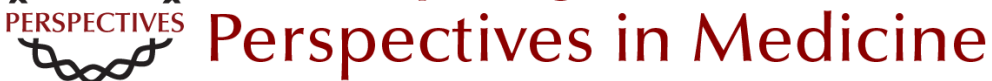

\section{Electromagnetic Regulation of Cell Activity}

Sarah A. Stanley and Jeffrey M. Friedman

Cold Spring Harb Perspect Med 2019; doi: 10.1101/cshperspect.a034322 originally published online September 24, 2018

\section{Subject Collection Bioelectronic Medicine}

Neural Control of Inflammation: Bioelectronic Medicine in Treatment of Chronic Inflammatory

Disease

Michael Eberhardson, Laura Tarnawski, Monica

Centa, et al.

Noninvasive Neuromodulation of Peripheral Nerve

Pathways Using Ultrasound and Its Current

Therapeutic Implications

Christopher Puleo and Victoria Cotero

Enteric Neuromodulation for the Gut and Beyond

Yogi A. Patel and Pankaj J. Pasricha

\section{Optogenetic Control of the Peripheral Nervous} System

Rui B. Chang

Closed-Loop Neuromodulation in Physiological and Translational Research Stavros Zanos

Electrical Impedance Methods in Neuromuscular Assessment: An Overview

Seward B. Rutkove and Benjamin Sanchez

Optogenetic Medicine: Synthetic Therapeutic

Solutions Precision-Guided by Light Haifeng Ye and Martin Fussenegger

Technobiology's Enabler: The Magnetoelectric Nanoparticle

Sakhrat Khizroev
Bioelectronic Medicine: From Preclinical Studies on the Inflammatory Reflex to New Approaches in Disease Diagnosis and Treatment

Valentin A. Pavlov, Sangeeta S. Chavan and Kevin J. Tracey

Vagus Nerve Stimulation and the Cardiovascular System

Michael J. Capilupi, Samantha M. Kerath and Lance B. Becker

Harnessing the Inflammatory Reflex for the Treatment of Inflammation-Mediated Diseases Yaakov A. Levine, Michael Faltys and David Chernoff

Recording and Decoding of Vagal Neural Signals Related to Changes in Physiological Parameters and Biomarkers of Disease

Theodoros P. Zanos

Restoring Movement in Paralysis with a

Bioelectronic Neural Bypass Approach: Current

State and Future Directions Chad E. Bouton

Bioelectronic Medicine--Ethical Concerns Samuel Packer, Nicholas Mercado and Anita Haridat

Use of Bioelectronics in the Gastrointestinal Tract Larry Miller, Aydin Farajidavar and Anil Vegesna

Vagus Nerve Stimulation at the Interface of BrainGut Interactions

Bruno Bonaz, Valérie Sinniger and Sonia Pellissier

For additional articles in this collection, see http://perspectivesinmedicine.cshlp.org/cgi/collection/ 